\title{
Sleep in Patients With Multiple Sclerosis
}

\author{
Tiffany J. Braley ${ }^{1}$
}

Published online: 15 April 2015

(C) Springer International Publishing AG 2015

\begin{abstract}
Sleep disorders are highly prevalent and consequential comorbidities among patients with multiple sclerosis (MS) yet remain notably under-recognized in clinical practice. Improved understanding of the specific clinical features of MS that may influence patients' risk for sleep disturbances or response to treatment could enhance opportunities to improve functional status and quality of life in persons with MS. This review aims to discuss current prevalence estimates, diseasespecific risk factors, and consequences of the most common sleep disorders experienced by patients with multiple sclerosis. Suggested methods to address MS-specific symptoms and comorbidities that may influence diagnostic and treatment strategies for these conditions are also highlighted.
\end{abstract}

Keywords Multiple sclerosis · MS · Fatigue · Sleepiness · Insomnia $\cdot$ Sleep-disordered breathing $\cdot$ Obstructive sleep apnea $\cdot$ OSA $\cdot$ Central sleep apnea $\cdot$ CSA $\cdot$ Restless legs syndrome $\cdot$ RLS $\cdot$ Willis-EkbomDisease (WED) · Brainstem . Spinal cord · Hypnotic $\cdot$ Diphenhydramine $\cdot$ Depression .

Pain · Spasticity

\section{Introduction}

Multiple sclerosis (MS) is an autoimmune disease that results in inflammation, myelin destruction, and axonal degeneration of the brain, spinal cord, and optic nerves. According to esti-

This article is part of the Topical Collection on Sleep and Neurological Disorders

Tiffany J. Braley

tbraley@med.umich.edu

1 Department of Neurology, Multiple Sclerosis and Sleep Disorders Centers, University of Michigan, C728 Med-Inn Building, 1500 E. Medical Center Dr., Ann Arbor, MI 48109, USA mates from the National Multiple Sclerosis Society, MS affects nearly half a million people in the USA and over 2.5 million people worldwide. Approximately $85 \%$ of patients will initially present with a relapsing-remitting course (relapsing-remitting MS), characterized by clearly defined episodes of neurological dysfunction (relapses, exacerbations, or "flare-ups") separated by periods of relative clinical stability (remissions). If left untreated, the majority patients with relapsing-remitting MS will eventually develop a gradual deterioration of neurologic function, usually in the form of paraparesis, hemiparesis, or dementia (secondary-progressive MS). A primary-progressive subtype occurs in a smaller percentage of patients, characterized by a slow deterioration in neurologic function from onset, without distinct relapses.

In addition to neurological morbidity, MS is associated with a disproportionately high prevalence of sleep disorders. In particular, sleep-disordered breathing, restless legs syndrome, and chronic insomnia are frequent contributors to poor functional outcomes in MS, as well as fatigue - one of the most common and debilitating symptoms experienced by MS patients $[1 \bullet, 2 \bullet, 3-9]$. Multiple sclerosis patients with suspected sleep problems are more likely than controls to emphasize problematic fatigue, tiredness, and lack of energy, as opposed to sleepiness [10]. Timely diagnosis and treatment of sleep disorders in MS patients offers a key opportunity to reduce fatigue, optimize general health, and enhance quality of life in this population, but an in-depth knowledge of MSrelated symptoms and pathology that may influence this approach is necessary for optimal management.

\section{Insomnia}

The International Classification of Sleep Disorders Diagnostic Manual Third Edition (ICSD-3) defines insomnia as "a persistent difficulty with sleep initiation, duration, consolidation, or quality that occurs despite the opportunity and 
circumstances for sleep, and results in some form of daytime impairment." [11] Impairments in daytime functioning related to insomnia are similar to many of the chronic symptoms experienced by MS patients and include fatigue, impaired concentration or memory, mood disturbances, excessive daytime sleepiness, behavioral problems, reduced motivation or energy, impaired social, family, academic, or occupational performance, proneness to errors, and concerns or dissatisfaction with sleep [11]. Also relevant to MS, the ICSD allows for specific clinical and pathophysiological subtypes of insomnia-including insomnia due to a medical condition or another mental disorder. Within this framework, at least 30$40 \%$ of patients with MS are at increased risk for insomnia $[1 \bullet, 2 \cdot 12,13]$.

Common triggers for insomnia in MS include chronic pain, urinary frequency, spasticity, anxiety, and depression. In a recent survey, a study of 195 clinically definite MS patients followed in a tertiary MS center, $46 \%$ were found to have moderate to severe clinical insomnia as defined by the Insomnia Severity Index (ISI) [1•]. Furthermore, $85 \%$ of patients studied endorsed at least one nocturnal symptom (pain, tingling, spasticity, feelings of restlessness, urinary urgency, anxiety, an inability to shut off the mind, or muscle twitching) to interfere with their ability to get a good night's sleep, with $54 \%$ of patients endorsing three or more of these symptoms. In this study, the number of nocturnal symptoms emerged as a strong predictor of ISI score, adjusting for other important clinical confounds [1•]. Similarly, in an anonymous survey study of 2375 community-dwelling adults with selfidentified MS [2•], $31 \%$ of those surveyed met ISI criteria for moderate to severe insomnia, while only $10 \%$ of these patients endorsed a formal diagnosis of insomnia by a physician, highlighting a large discrepancy between insomnia prevalence and recognition in this population.
Given these findings, it is recommended that all MS patients who endorse daytime impairment or express concerns about prolonged sleep latency, fragmented sleep, unrefreshing sleep, or early terminal awakenings be evaluated for insomnia. This can be accomplished with a systematic approach that integrates a thorough assessment of MSspecific comorbidities and symptoms with the sleep history. If not initially volunteered, a thorough review of daytime symptoms should also be conducted to characterize any associated daytime distress or impairment. As with non-MS patients, further inquiries should be directed toward external factors or habits that may interfere with sleep hygiene.

A thorough medication assessment (that includes over-thecounter medications) is also a key component of the insomnia assessment, with special focus on centrally acting agents such as selective serotonin reuptake inhibitors, stimulants and wake-promoting agents, and over-the-counter antihistamines, as these therapies are frequently used in patients with MS [13, 14]. Corticosteroids, which are typically used for short periods to treat MS exacerbations may also contribute to symptoms of insomnia in the short term (Table 1) [15]. Patients who use beta-interferon disease-modifying therapies for their MS are also at increased risk for sleep disturbances. This risk may be increased in patients who administer their medication during the evening hours [16].

Treatment of insomnia in patients with MS should start with amelioration of any precipitating causes, either prior to or in tandem with first-line therapies. Medications or substances that may contribute to insomnia should be minimized, if possible. Wake-promoting agents and stimulants should be reserved for the early hours of the day. Chronic symptoms such as nocturnal spasticity, neuropathic pain, or nocturia should be addressed. If neuropathic pain or spasticity are contributing factors, effective medications that also have sedating
Table 1 Factors that may exacerbate or influence vulnerability to common sleep disorders in MS patients

\begin{tabular}{lll}
\hline Insomnia & Sleep-disordered breathing & Restless legs syndrome \\
\hline Medications & Medications & Medications/substances \\
- Corticosteroids & - Opioids & - 1st-generation antihistamines \\
- Stimulants/wake-promoting agents & - Benzodiazepines & - Dopamine antagonists \\
- SSRI's & - Antispasmodics & - Lithium \\
$\quad$ - Beta-interferons & Brainstem dysfunction & - Alcohol \\
Depression/anxiety & Increased disability & - Tobacco \\
Pain & Progressive MS subtype & - Caffeine \\
Spasticity & General risk factors & Primary progressive MS \\
Nocturia & - Age $>50$ years & Increased disability \\
Other sleep disorders & - Obesity & Cervical spinal cord lesions \\
- Sleep-disordered breathing & - Increased neck circumference & Iron deficiency \\
- Restless legs syndrome & - Crowded oropharyngeal outlet & Family history \\
$\quad$ - Periodic leg movements of sleep & - Retrognathia/micrognathia & \\
Poor sleep hygiene & & \\
\hline
\end{tabular}


properties (such as tricyclic antidepressants or antispasmodics, respectively) may be reasonable first options.

If comorbid symptoms are not significant contributing factors, psychological and behavioral therapies should be considered. Cognitive behavioral therapy for insomnia (CBT-I) aims to target maladaptive thoughts and behaviors that can perpetuate insomnia. The benefits of CBT-I are well studied [17-20], making it an ideal treatment approach for patients with MS, particularly in patients with comorbid depression. Depression affects approximately $50 \%$ of MS patients at some point during the disease course and shares a bidirectional relationship with insomnia [21]. Recent data show that rates of insomnia are higher in depressed MS patients than non-depressed MS patients and suggest that insomnia management should commence beyond the treatment of the underlying comorbid psychiatric disorder [22]. Cognitive behavioral therapy may also be an effective treatment modality for MS-related fatigue [23].

For patients who are not responsive to more conservative strategies, pharmacological therapies may be necessary. In this case, as with non-MS patients, selection of an appropriate agent should be guided in part by the drug's half-life, as well as the patient's intolerances and comorbidities. Benzodiazepine receptor agonists, melatonin receptor agonists, and newer orexin receptor antagonists are all potentially useful therapies for MS patients with insomnia. Although the affordability and widespread availability of over-the-counter antihistaminecontaining products make them a popular treatment for insomnia, it is recommended that these agents be avoided in patients with MS. Recent evidence suggests that nocturnal antihistamine use is independently associated with increased daytime fatigue in MS patients [13].

\section{Sleep-Disordered Breathing}

Obstructive sleep apnea (OSA) is characterized by repeated episodes of upper airway obstruction and hypoxia during sleep. According to recent reports, up to $21 \%$ of MS patients may carry a formal diagnosis of OSA [1•]. Furthermore, a much higher proportion of MS patients (38-56\%) may be at risk for OSA, based on two recently published studies $[1 \bullet, 2 \bullet]$ that evaluated MS patients with a validated OSA screening tool [24].

While reasons behind this relationship require further exploration, underlying neuroanatomical and immunological features of MS may be contributing factors. Central nervous system disorders that disrupt brainstem pathways responsible for the maintenance of nocturnal airway patency have the potential to impair nocturnal respiration [25-28]. In a previous study, MS subjects referred for overnight polynsomnography - and particularly those patients with MRI evidence of brainstem involvementwere found to have more severe OSA than control subjects without MS [29•]. Among the MS subjects in this study, progressive subtypes of MS and MS immunomodulatory therapy use also predicted apnea severity. Immunomodulatory therapy use in particular emerged as a strong predictor of reduced apnea severity.

Central nervous disorders that affect brainstem respiratory generators, including MS, may also be associated with an increased risk for central sleep apnea [28, 29॰]. In addition to alterations in control of nocturnal airway patency, patients with evidence of brainstem dysfunction may be more vulnerable to impairments in autonomic nocturnal respiratory control, due to damage of key brainstem respiratory centers located in the pons and medulla [30, 31].

Multiple sclerosis patients who have a diagnosis of OSA and those at elevated risk for OSA have increased fatigue levels compared to undiagnosed or low-risk patients $[1 \bullet, 2 \bullet$, $3,4]$. Obstructive sleep apnea is also a predictor of diminished quality life in MS [6], and preliminary research suggests that apnea severity may correlate with cognitive impairment in MS [32].

Given the detrimental effects of OSA in this population, early identification and treatment of sleep-disordered breathing is critical. All MS patients should be asked about common symptoms of OSA, such as snoring, gasping or choking upon awakening, non-restorative sleep, excessive daytime hypersomnolence or fatigue, cognitive disturbances, or nighttime arousals. Clinical signs of brainstem dysfunction (such as dysarthria or dysphagia) or the presence of brainstem lesions on MRI should also alert clinicians to consider full-night polysomnogram (PSG) to rule out OSA or CSA.

Positive airway pressure therapy improves fatigue as well as sleepiness in non-MS patients [33] and is the gold standard treatment for MS patients with OSA. In select cases, oral appliances may also be considered. Although surgical approaches for OSA are also available, the expected neurological dysfunction as a possible exacerbating factor of OSA among MS patients usually makes surgery less attractive as a definitive treatment.

\section{Restless Legs Syndrome}

Restless legs syndrome (RLS, also known as Willis-Ekbom disease) is characterized by an urge to move the legs, often accompanied with restlessness or an uncomfortable sensation that is exacerbated by rest and inactivity, has a tendency to occur in the evening or before bedtime, and is relieved with movement $[34,35]$. This condition affects approximately $10 \%$ of the general population [36]. Restless legs syndrome is classified as idiopathic or primary if no other cause can be identified, or secondary (symptomatic) if associated with another comorbid condition such as MS.

While the exact mechanism underlying primary RLS is unknown, dysfunction of brain circuits that require the neurotransmitter dopamine have been implicated in the pathogenesis of RLS [37]. As iron is a component of the enzyme 
tyrosine dehydroxylase (responsible for the rate-limiting step in dopamine synthesis), it seems intuitive that impaired iron metabolism is also thought to contribute to the pathogenesis of RLS. This hypothesis is supported clinically by lower serum and CSF ferritin levels in patients with idiopathic RLS [38] and low brain iron stores in RLS patients in MRI and autopsy studies [39, 40]. A genetic etiology for RLS is favored for younger patients who experience signs of idiopathic RLS before age 40 [41-43].

Other investigators suggest that RLS may be caused by dysfunction of downstream dopaminergic pathways, namely diencephalospinal and reticulospinal pathways [44] that project from the brain to the spinal cord. These pathways are thought to be responsible for the suppression of excessive sensory inputs and autonomic output and are susceptible to damage from a variety of pathologic processes, which may explain the increased prevalence of RLS in MS and other conditions that can affect the spinal cord [45-47]. Restless legs syndrome is approximately three times more common in MS as compared to the general population $[45,48,49]$. In a pivotal study of prospectively surveyed MS subjects, $32 \%$ met criteria for RLS. Clinical features of MS that predicted RLS in this study included primary progressive MS subtype and higher level of neurological disability [45]. Similar prevalence estimates were identified in two recent survey studies of MS patients $[1 \bullet, 2 \bullet]$. In a multicenter case-control study of 861 MS subjects [50], $19 \%$ experienced frequent RLS symptoms at least twice per week (lower frequencies were not included in this estimate). In this study, RLS was again more common in patients with increased disability. Studies of radiographic correlates of RLS in MS subjects suggest that cervical cord damage may also be an independent risk factor for RLS, highlighting a potentially important relationship between RLS and dopaminergic spinal cord projections [46, 50].

Patients with RLS most often describe an uncomfortable sensation in their legs (or less frequently, the arms) that interferes with their ability to rest [34]. Many descriptors have been used to define this sensation, including creeping, crawling, itching, burning, tightening, or tingling. Others will describe this sensation as painful [51]. While heterogeneity in descriptions is common, the diagnosis of RLS requires that the symptoms of discomfort are not better accounted for by another medical or behavioral condition. The differential diagnosis for RLS is broad and includes several other symptoms that may be particularly common in MS, such as cramping, spasticity, or neuropathic pain [52]. While distinguishing between these symptoms can be challenging, reports of a circadian predilection and relief with movement is more suggestive of RLS, while painful, involuntary muscle contractions, or symptoms not relieved by movement may be more suggestive of spasticity or neuropathic pain, respectively.
To date, there are no MS-specific treatment guidelines for the management of RLS, but in the author's experience, treatment should be individualized and tailored to the patient's symptoms and other comorbid conditions whenever possible. As in non-MS patients, the treatment of RLS in MS patients ranges from conservative to pharmacological approaches, which can be employed alone or in combination. Conservative approaches include the removal of various agents known to exacerbate RLS such as alcohol, tobacco, and caffeine. Serum ferritin levels should be assessed and iron supplementation should be implemented for ferritin levels less than $50 \mathrm{ng} / \mathrm{ml}$. An evaluation of the patient's medication list is also recommended, with minimization of medications that can cause or worsen RLS such as dopamine antagonists, lithium, selective serotonin reuptake inhibitors, and tricyclic antidepressants. Multiple sclerosis patients should also be screened for antihistamine use, as the use of over-the-counter antihistamine hypnotic use is common in MS and is associated with a higher prevalence of RLS [13].

Dopamine agonists (rotigotine, pramipexole, and ropinirole) and the alpha-2-delta ligand gabapentin enacarbil are the only FDA-approved first-line medications for moderate to severe RLS and are reasonable pharmacologic choices for MS patients with RLS [53, 54]. Common side effects of dopaminergic agents - including nausea, hypotension, hallucinations, dyskinesias, and increased risks of impulse control behaviors - should be discussed with the patient before initiation and should be reconsidered in MS patients with concomitant autonomic dysfunction. Patients should also be followed closely for signs of augmentation, a phenomenon that involves worsening of RLS symptoms earlier in the day with geographic spread to other body regions over time [55]. If dopaminergic agents are inappropriate or poorly tolerated, other drug classes should be considered.

Several anticonvulsants, including gabapentin, carbamazepine, and most recently pregabalin [56••], are reasonable pharmacologic alternatives for patients who cannot tolerate or have failed dopaminergic agents and may be particularly ideal choices for MS patients who are more likely to suffer from concomitant conditions such as neuropathic pain or seizures.

Benzodiazepines have also demonstrated therapeutic effect in RLS, although data supporting these agents are less robust and confounded by other benzodiazepine effects on sleep [57]. Given the long half-life of some benzodiazepines (which may cause next day carry-over effects), their use is generally not recommended in MS patients who already suffer from daytime fatigue. Various opioid agents, including oxycodone and methadone, may be of benefit in selected individuals whose RLS symptoms may be refractory to first-line agents, but the addiction potential and side effect profile associated with opioids limit their use, and opioids are discouraged in MS patients. 


\section{Conclusion}

Insomnia, sleep-disordered breathing, and restless legs syndrome are common and yet frequently under-recognized conditions in patients with MS. Providers caring for MS patients should maintain a low threshold to screen for sleep disorders, given their high prevalence and serious consequences. Increased efforts are needed to identify sleep disorders among MS patients who are most vulnerable, and a systematic approach is necessary to distinguish sleep disturbances from other comorbid conditions in MS. Improved recognition and treatment of sleep disturbances have the potential to significantly improve MS patients' functional outcomes and quality of life.

Acknowledgment Activities associated with this review received no specific grant from any funding agency in the public, commercial, or not-for-profit sectors.

\section{Compliance With Ethics Guidelines}

Conflict of Interest Tiffany J. Braley has received grants from the National Multiple Sclerosis Society, American Sleep Medicine Foundation, and the Michigan Translational Research and Commercialization for Life Sciences Program (U-M MTRAC). Dr. Braley has served as site P.I. on several industry-sponsored studies at the University of Michigan but received no direct compensation for this work and is also named in a provisional patent concerning treatment for obstructive sleep apnea.

Human and Animal Rights and Informed Consent This article does not contain any studies with human or animal subjects performed by any of the authors.

\section{References}

Papers of particular interest, published recently, have been highlighted as:

- Of importance

•- Of major importance

1. Braley TJ, Segal BM, Chervin RD. Obstructive sleep apnea and fatigue in patients with multiple sclerosis. J Clin Sleep Med. 2014;10(2):155-62. Highlights the prevalence of OSA, elevated OSA risk, insomnia, and other sleep disturbances in MS and their relationship to fatigue.

2. Brass SD, Li CS, Auerbach S. The underdiagnosis of sleep disorders in patients with multiple sclerosis. J Clin Sleep Med. 2014;10(9):1025-31. Demonstrates the discrepancy between symptoms and clinical regcognition of sleep disorders in MS.

3. Veauthier C, Radbruch H, Gaede G, et al. Fatigue in multiple sclerosis is closely related to sleep disorders: a polysomnographic cross-sectional study. Mult Scler. 2011;17(5):613-22.

4. Kaminska M, Kimoff RJ, Benedetti A, et al. Obstructive sleep apnea is associated with fatigue in multiple sclerosis. Mult Scler. 2012;18(8):1159-69.

5. Newland PK, Naismith RT, Ullione M. The impact of pain and other symptoms on quality of life in women with relapsingremitting multiple sclerosis. J Neurosci Nurs. 2009;41(6):322-8.
6. Trojan DA, Kaminska M, Bar-Or A, et al. Polysomnographic measures of disturbed sleep are associated with reduced quality of life in multiple sclerosis. J Neurol Sci. 2012;316(1-2):158-63.

7. Krupp LB, Alvarez LA, LaRocca NG, Scheinberg LC. Fatigue in multiple sclerosis. Arch Neurol. 1988;45(4):435-7.

8. Krupp L. Fatigue is intrinsic to multiple sclerosis (MS) and is the most commonly reported symptom of the disease. Mult Scler. 2006;12(4):367-8.

9. Lerdal A, Celius EG, Krupp L, Dahl AA. A prospective study of patterns of fatigue in multiple sclerosis. Eur J Neurol. 2007;14(12): 1338-43.

10. Braley TJ, Chervin RD, Segal BM. Fatigue, tiredness, lack of energy, and sleepiness in multiple sclerosis patients referred for clinical polysomnography. Mult Scler Int. 2012;2012:673936.

11. American Academy of Sleep Medicine. International Classification of Sleep Disorders (ICSD-3): Diagnostic and Coding Manual. 3rd ed., ed. I. American Academy of Sleep Medicine. Darien. 2014.

12. Tachibana N, Howard RS, Hirsch NP, Miller DH, Moseley IF, Fish D. Sleep problems in multiple sclerosis. Eur Neurol. 1994;34(6): 320-3.

13. Braley TJ, Segal BM, Chervin RD. Hypnotic use and fatigue in multiple sclerosis. Sleep Med. 2015;16(1):131-7.

14. Byerley WF, Reimherr FW, Wood DR, Grosser BI. Fluoxetine, a selective serotonin uptake inhibitor, for the treatment of outpatients with major depression. J Clin Psychopharmacol. 1988;8(2):112-5.

15. Shaygannejad V, Ashtari F, Alinaghian M, Norouzi R, Salari M, Fatehi F. Short-term safety of pulse steroid therapy in multiple sclerosis relapses. Clin Neuropharmacol. 2013;36(1):1-3.

16. Nadjar Y, Coutelas E, Prouteau P, et al. Injection of interferon-beta in the morning decreases flu-like syndrome in many patients with multiple sclerosis. Clin Neurol Neurosurg. 2011;113:316-22

17. Edinger JD, Means MK. Cognitive-behavioral therapy for primary insomnia. Clin Psychol Rev. 2005;25(5):539-58.

18. Edinger JD, Wohlgemuth WK, Radtke RA, Marsh GR, Quillian RE. Cognitive behavioral therapy for treatment of chronic primary insomnia: a randomized controlled trial. JAMA. 2001;285(14): 1856-64.

19. Sivertsen B, Omvik S, Pallesen S, et al. Cognitive behavioral therapy vs zopiclone for treatment of chronic primary insomnia in older adults: a randomized controlled trial. JAMA. 2006;295(24):28518.

20. Morin CM, Vallieres A, Guay B, et al. Cognitive behavioral therapy, singly and combined with medication, for persistent insomnia: a randomized controlled trial. JAMA. 2009;301(19):2005-15.

21. Figved N, Klevan G, Myhr KM, et al. Neuropsychiatric symptoms in patients with multiple sclerosis. Acta Psychiatr Scand. 2005;112(6):463-8.

22. Baron KG, Corden M, Jin L, Mohr DC. Impact of psychotherapy on insomnia symptoms in patients with depression and multiple sclerosis. J Behav Med. 2011;34(2):92-101.

23. van Kessel K, Moss-Morris R, Willoughby E, Chalder T, Johnson $\mathrm{MH}$, Robinson E. A randomized controlled trial of cognitive behavior therapy for multiple sclerosis fatigue. Psychosom Med. 2008;70(2):205-13.

24. Chung F, Subramanyam R, Liao P, Sasaki E, Shapiro C, Sun Y. High STOP-Bang score indicates a high probability of obstructive sleep apnoea. Br J Anaesth. 2012;108(5):768-75.

25. Brown DL, McDermott M, Mowla A, et al. Brainstem infarction and sleep-disordered breathing in the BASIC sleep apnea study. Sleep Med. 2014;15(8):887-91.

26. Fogel RB, Trinder J, White DP, et al. The effect of sleep onset on upper airway muscle activity in patients with sleep apnoea versus controls. J Physiol. 2005;564(Pt 2):549-62.

27. Jordan AS, White DP. Pharyngeal motor control and the pathogenesis of obstructive sleep apnea. Respir Physiol Neurobiol. 2008;160(1):1-7. 
28. Losurdo A, Dittoni S, Testani E, et al. Sleep disordered breathing in children and adolescents with Chiari malformation type I. J Clin Sleep Med. 2013;9(4):371-7.

29. Braley TJ, Segal BM, Chervin RD. Sleep-disordered breathing in multiple sclerosis. Neurology. 2012;79(9):929-36. First of its kind study to address the clinical and radiographic risk factors for sleep disordered breathing among patients with MS.

30. Dyken ME, Im KB. Obstructive sleep apnea and stroke. Chest. 2009;136(6):1668-77.

31. Dyken ME, Afifi AK, Lin-Dyken DC. Sleep-related problems in neurologic diseases. Chest. 2012;141(2):528-44.

32. Braley TJ, Kratz AL, Kaplish N, Chervin RD. Cognitive dysfunction in multiple sclerosis is associated with obstructive sleep apnea. Sleep. 2014;37(abstract supplement):A241.

33. Chotinaiwattarakul W, O'Brien LM, Fan L, Chervin RD. Fatigue, tiredness, and lack of energy improve with treatment for OSA. J Clin Sleep Med. 2009;5(3):222-7.

34. Allen RP, Picchietti D, Hening WA, Trenkwalder C, Walters AS, Montplaisi J. Restless legs syndrome: diagnostic criteria, special considerations, and epidemiology. A report from the restless legs syndrome diagnosis and epidemiology workshop at the National Institutes of Health. Sleep Med. 2003;4(2): 101-19.

35. Hening W, Allen RP, Tenzer P, Winkelman JW. Restless legs syndrome: demographics, presentation, and differential diagnosis. Geriatrics. 2007;62(9):26-9.

36. Rothdach AJ, Trenkwalder C, Haberstock J, Keil U, Berger K. Prevalence and risk factors of RLS in an elderly population: the MEMO study. Memory and Morbidity in Augsburg Elderly. Neurology. 2000;54(5):1064-8.

37. Cervenka S, Palhagen SE, Comley RA, et al. Support for dopaminergic hypoactivity in restless legs syndrome: a PET study on D2receptor binding. Brain. 2006;129(Pt 8):2017-28.

38. Earley CJ, Connor JR, Beard JL, Clardy SL, Allen RP. Ferritin levels in the cerebrospinal fluid and restless legs syndrome: effects of different clinical phenotypes. Sleep. 2005;28(9):1069-75.

39. Allen RP, Barker PB, Wehrl F, Song HK, Earley CJ. MRI measurement of brain iron in patients with restless legs syndrome. Neurology. 2001;56(2):263-5.

40. Connor JR, Boyer PJ, Menzies SL, et al. Neuropathological examination suggests impaired brain iron acquisition in restless legs syndrome. Neurology. 2003;61(3):304-9.

41. Pichler I, Hicks AA, Pramstaller PP. Restless legs syndrome: an update on genetics and future perspectives. Clin Genet. 2008;73(4):297-305.

42. Winkelmann J, Polo O, Provini F, et al. Genetics of restless legs syndrome (RLS): state-of-the-art and future directions. Mov Disord. 2007;22(18):S449-458.
43. Winkelmann J, Schormair B, Lichtner P, et al. Genome-wide association study of restless legs syndrome identifies common variants in three genomic regions. Nat Genet. 2007;39(8):1000-6.

44. Frauscher B, Loscher W, Hogl B, Poewe W, Kofler M. Auditory startle reaction is disinhibited in idiopathic restless legs syndrome. Sleep. 2007;30(4):489-93.

45. Manconi M, Fabbrini M, Bonanni E, et al. High prevalence of restless legs syndrome in multiple sclerosis. Eur J Neurol. 2007;14(5):534-9.

46. Manconi M, Rocca MA, Ferini-Strambi L, et al. Restless legs syndrome is a common finding in multiple sclerosis and correlates with cervical cord damage. Mult Scler. 2008;14(1):86-93.

47. Telles SC, Alves RC, Chadi G. Periodic limb movements during sleep and restless legs syndrome in patients with ASIA A spinal cord injury. J Neurol Sci. 2011;303(1-2):119-23.

48. Auger C, Montplaisir J, Duquette P. Increased frequency of restless legs syndrome in a French-Canadian population with multiple sclerosis. Neurology. 2005;65(10):1652-3.

49. Deriu M, Cossu G, Molari A, et al. Restless legs syndrome in multiple sclerosis: a case-control study. Mov Disord. 2009;24(5): 697-701.

50. Italian RSG, Manconi M, Ferini-Strambi L, et al. Multicenter casecontrol study on restless legs syndrome in multiple sclerosis: the REMS study. Sleep. 2008;31(7):944-52.

51. Bassetti CL, Mauerhofer D, Gugger M, Mathis J, Hess CW. Restless legs syndrome: a clinical study of 55 patients. Eur Neurol. 2001;45(2):67-74.

52. Hening WA, Allen RP, Washburn M, Lesage SR, Earley CJ. The four diagnostic criteria for restless legs syndrome are unable to exclude confounding conditions ("mimics"). Sleep Med. 2009;10(9):976-81.

53. Littner MR, Kushida C, Anderson WM, et al. Practice parameters for the dopaminergic treatment of restless legs syndrome and periodic limb movement disorder. Sleep. 2004;27(3):557-9.

54. Wilt TJ, Macdonald R, Ouellette J, et al. Pharmacologic therapy for primary restless legs syndrome: a systematic review and meta-analysis. JAMA Intern Med. 2013;173(7):496-505.

55. Allen RP, Earley CJ. Augmentation of the restless legs syndrome with carbidopa/levodopa. Sleep. 1996;19(3):205-13.

$56 . \bullet$ Allen RP, Chen C, Garcia-Borreguero D, et al. Comparison of pregabalin with pramipexole for restless legs syndrome. N Engl J Med. 2014;370(7):621-31. Clinical trial that highlights the utility of pregabalin as an alternative treatment to dopaminergic agents for RLS.

57. Chesson Jr AL, Wise M, Davila D, et al. Practice parameters for the treatment of restless legs syndrome and periodic limb movement disorder. An American Academy of Sleep Medicine Report. Standards of Practice Committee of the American Academy of Sleep Medicine. Sleep. 1999;22(7):961-8. 